\title{
CIANOBACTÉRIAS EM ESTEIRAS MICROBIANAS COLOFORMES DA LAGOA PITANGUINHA, RIO DE JANEIRO, BRASIL
}

\author{
CYNTHIA MOREIRA DAMAZIO \\ Laboratório de Taxonomia Algal, UNIRIO, Avenida Pasteur, 458, 22.290-240, Rio de Janeiro, Brasil.acdamazio@uol.com.br \\ LOREINE HERMIDA SILVA E SILVA \\ Núcleo de Geomicrobiologia, UNIRIO, Avenida Pasteur, 458, 22.290-240, Rio de Janeiro, Brasil. loreineh@unirio.br
}

\begin{abstract}
RESUMO - O objetivo deste estudo foi a identificação das cianobactérias constituintes das esteiras coloformes formadas no fundo da lagoa Pitanguinha, restinga de Massambaba, no município de Araruama, estado do Rio de Janeiro (coordenadas $22^{\circ} 55^{\prime} 42^{\prime \prime} / 22^{\circ} 56^{\prime} 00^{\prime \prime S}, 42^{\circ} 20^{\prime} 45^{\prime \prime} / 42^{\circ} 21^{\prime} 30^{\prime \prime} \mathrm{W}$ ). Para tanto, foram realizadas coletas mensais, entre os meses de março de 2003 a fevereiro de 2004. A esteira coloforme se apresentou estratificada, com uma camada superficial onde predominam as formas filamentosas e uma mais profunda, onde dominam as formas esféricas, comprovando o alto grau de maturação. Entre seus constituintes, 24 tipos de cianobactérias foram identificados, incluídos nas famílias Chroococcaceae, a mais abundante, Synechococcaceae, Phormidiaceae, Oscillatoriaceae, Pseudanabaenaceae, Schizothricaceae e Entophysalidaceae. Esta diversidade atesta a resistência destes organismos aos ambientes inóspitos e pode ser útil na interpretação de depósitos similares no registro fóssil.
\end{abstract}

Palavras-chave: Esteiras microbianas coloformes, cianobactérias, lagoa Pitanguinha.

\begin{abstract}
CYANOBACTERIA IN COLOFORM MICROBIAL MATS FROM THE PITANGUINHA LAGOON, RIO DE JANEIRO STATE, BRAZIL. This study aims the identification of the cyanobacteria that constitutes the coloform microbial mats find in the bottom of the Pitanguinha Lagoon, Massambaba shoal, in the municipality of Araruama, Rio de Janeiro state (coordinates $22^{\circ} 55^{\prime} 42^{\prime \prime} / 22^{\circ} 56^{\prime} 00^{\prime \prime} \mathrm{S}, 42^{\circ} 20^{\prime} 45^{\prime \prime} / 42^{\circ} 21^{\prime} 30^{\prime \prime} \mathrm{W}$ ). It was based on monthly sampling made between March of 2003 and February of 2004. The coloform mat is stratified, with a surface layer dominated by filamentous forms, and the deepest by spherical forms, which attest the mat maturity. A total of 24 taxa of cyanobacteria were identified and included in the families Chroococcaceae, Synechococcaceae, Phormidiaceae, Oscillatoriaceae, Pseudanabaenaceae, Schizothricaceae and Entophysalidaceae. The mat diversity informs about the resistance of cyanobacteria to stressing environments and could be useful in the interpretation of similar deposits in the fossil record.
\end{abstract}

Key words: Coloform microbial mats, cyanobacteria, Pitanguinha Lagoon.

\section{INTRODUÇÃO}

Lagoas costeiras podem ser definidas como depressões contendo água salobra ou salgada, localizadas em áreas litorâneas. Formam-se em regiões situadas abaixo do nível do mar e dele são separadas por cordões litorâneos (Weber, 2001). A hipersalinidade em lagoas costeiras pode resultar de mudanças climáticas, de registros de ciclos globais e do impacto antrópico (Turcq, 2000). Como estes ambientes ocupam áreas de transição entre o oceano e os continentes, acumulam material de ambas as áreas e atuam como regiões de acumulação e filtração (Patchineelan \& Naidu, 1995).

As condições físicas e químicas das lagoas variam significativamente, sendo freqüente a formação de um ambiente biológico de alto estresse. Desta forma, é comum possuírem uma biota menos diversificada do que o ambiente marinho (Lemos, 1996). Nos ambientes lagunares, os microorganismos exercem um importante papel, especialmente na decomposição e na mineralização da matéria orgânica produzida (Abreu \& Odebrecht, 2000).

O litoral fluminense se caracteriza pela presença de um grande número de lagoas (Barroso, 1987). Na bacia hidrográfica da lagoa de Araruama e em seu entorno, em especial na restinga de Massambaba, encontram-se várias lagoas, tais como a Vermelha, a da Pitanguinha, a de Pernambuco e a Azul (Primo \& Bizerril, 2002).

As cianobactérias foram um dos primeiros seres vivos a habitar o planeta, há cerca de 3,5 bilhões de anos. Atualmente, esteiras e estromatólitos são produzidas por comunidades microbianas compostas principalmente por cianobactérias (Golubic, 1976), organismos que, por meio da fotossíntese, obtêm energia para crescer e se reproduzir (Nascimento, 1999). 
O objetivo deste estudo foi a identificação das cianobactérias constituintes das esteiras coloformes encontradas no assoalho da lagoa Pitanguinha. A ausência de estudos sobre as estruturas estromatolíticas aí presentes, garante a relevância deste estudo e complementa as pesquisas que vem sendo realizadas nas demais lagoas e brejos do complexo lagunar de Araruama.

\section{ÁREA DE ESTUDO}

\section{Condicionantes climáticos}

A lagoa Pitanguinha possui aproximadamente $0,55 \mathrm{~km}^{2}$, com $1,5 \mathrm{~km}$ de comprimento e $0,75 \mathrm{~km}$ de largura (Figura 1A). Localiza-se entre as latitudes de $22^{\circ} 55^{\prime} 42^{\prime \prime} \mathrm{e}$ $22^{\circ} 56^{\prime} 00^{\prime \prime} \mathrm{S}$; e as longitudes de $42^{\circ} 20^{\prime} 45^{\prime \prime}$ e $42^{\circ} 21^{\prime} 30^{\prime \prime} \mathrm{W}$, na restinga de Massambaba, município de Araruama, Estado do Rio de Janeiro. Mais de dois terços de seu perímetro é rodeado por salinas e, internamente, marnéis a segmentam em várias porções. Canais a ligam com a lagoa de Araruama que, por sua vez, comunica-se com o mar (Primo \& Bizerril, 2002).

O clima na região é semi-árido, com grande déficit no balaço precipitação-evaporação (Silva e Silva et al., 2003a), a temperatura média do ar atmosférico varia entre $19^{\circ}$ e $31^{\circ} \mathrm{Ce}$ o índice pluviométrico anual é em torno de $1.000 \mathrm{~mm}$, com evaporação de $1.400 \mathrm{~mm}$ (Silva e Silva et al., 2003b).

Os ventos têm papel importante na mistura e na circulação das águas, com ventos de nordeste atuando no decorrer do ano, com velocidades variadas e médias de $6 \mathrm{~m} / \mathrm{s}$ no período de primavera/verão (Fonseca, 2002). Os ventos alíseos de sudeste dominam a circulação atmosférica, garantindo um clima ensolarado, com pouca chuva (Lopes, 1988). Devido à baixa profundidade da lagoa, a ação dos ventos ocasiona a agitação das águas e arrasta partículas finas em suspensão, produzindo uma homogeneização dos sedimentos depositados no fundo (Anjos, 1999).

\section{Geologia}

Durante o Quaternário, o litoral do Estado do Rio de Janeiro se caracterizou pelas flutuações do nível relativo do mar. Essas oscilações foram de fundamental importância na evolução das áreas costeiras no Brasil (Vasconcelos, 1988). A lagoa Pitanguinha tem sua formação relacionada a duas transgressões marinhas sucessivas, que ocasionaram o aparecimento de duas séries de cordões litorâneos de idades diferentes, responsáveis pelo fechamento da lagoa. Estes cordões foram alimentados por depósitos continentais acumulados sob a forma de glacis, durante as fases regressivas (Silva e Silva et al., 2003a).

Entre os dois sistemas de lagoas da região (interno e externo), a lagoa Pitanguinha situa-se no mais externo, formado por lagoas e brejos (Turcq et al., 1999), resultantes do último episódio de transgressão holocênica, iniciado há 7.000 anos (Anjos, 1999). A datação por ${ }^{14} \mathrm{C}$ das conchas e sambaquis da barreira arenosa e as condições paleogeográficas indicam uma idade entre 4.200 a 3.800 anos A.P. (Lopes, 1988). As areias são quartzosas, com grãos variando de muito finos a grossos, e de subangulosos à subarredondados (Silva e Silva et al., 2003a).

\section{ESTEIRAS MICROBIANAS}

As esteiras microbianas podem ser chamadas de esteiras algais, esteiras algálicas (algal mats), esteiras estromatolíticas, esteiras de cianobactérias e estromatólitos em potencial. Esta pluralidade de terminologias ocorre devido à predominância, em sua composição, de espécies de cianobactérias, responsáveis pela formação da estrutura sedimentar laminada. Estão entre as formas mais antigas de comunidade microbiana, sendo registradas desde cerca de 3,5 bilhões de anos atrás (Urmeneta \& Navarrete, 2000).

Segundo Van Germerdem (1993), as esteiras são estruturas organo-sedimentares laminadas que se desenvolvem sobre superfícies sólidas. Freqüentemente, possuem gradientes saturados de oxigênio e de sulfeto. Esteiras recentes têm sido encontradas principalmente em ambientes marinhos costeiros salinos ou hipersalinos. São produzidas como resultado da interação ambiental e da comunidade bentônica (Batchelos et al., 2000). As cianobactérias, agentes biológicos componentes destas esteiras, arranjam-se de forma peculiar e são responsáveis pela retenção e aprisionamento do sedimento que as compõem. Estes arranjos podem formar tufos, retículos, bem como se apresentar enovelados, o que origina os diferentes modelos (Silva e Silva et al., 2003a).

As esteiras são comumente encontradas sobre o substrato de areia siliciclástica em diversas regiões do mundo (Schieber, 1998). Podem se distribuir no perfil da costa desde a região de inframaré até a de supramaré e são mais visíveis durante os períodos de baixa precipitação pluvial. Dependendo da estação do ano, podem ficar completamente submersas. O desenvolvimento das esteiras microbianas está relacionado com as espécies de cianobactérias esféricas e filamentosas que formam os primeiros depósitos orgânicos, posteriormente degradados e modificados pela atividade bacteriana subseqüente (Silva e Silva, 2002).

As populações de cianobactérias se dispõem em pequenas camadas horizontais, que podem variar de alguns milímetros até alguns centímetros (Urmeneta \& Navarrete, 2000). A estratificação interna da esteira é provocada pela variação dos fatores ambientais. Na lagoa Pitanguinha há uma zona eufótica, onde a matéria orgânica é produzida por meio da fotossíntese. Porém, algumas cianobactérias apresentam baixa produção fotossintética nesta parte iluminada e só alcançam sua máxima produção entre 1 e $4 \mathrm{~mm}$ abaixo da superfície (Silva e Silva, 2002).

A acumulação das esteiras depende das cianobactérias filamentosas que aprisionam e unem os sedimentos (Noffke et al., 2001). Este crescimento se dá pelo acréscimo de lâminas de sedimentos aprisionados pela precipitação do carbonato, resultante da atividade dos organismos microbianos (Altermann \& Kazmierezak, 2003). Devido à sedimentação rápida, as cianobactérias são soterradas e a formação de uma nova colônia pode ocorrer na superfície (Silva e Silva et al., 2002). A umectação é importante nas esteiras, sendo a 
capacidade de reter água devida aos sedimentos incluídos em seus interstícios (Silva e Silva et al., 2003a).

Assim como os diferentes modelos de esteira, a composição taxonômica das comunidades microbianas está relacionada com as condições do meio. Deste modo, podem constituir importante ferramenta geológica nas reconstruções paleoambientais e taxonômicas.

\section{MATERIAIS E MÉTODOS}

Para a escolha das cinco estações de coleta foram estabelecidas as áreas na lagoa Pitanguinha onde ocorriam faixas de areia capazes de permitir a formação das esteiras. No presente caso, uma distância de aproximadamente $100 \mathrm{~m}$ separa cada uma delas. As amostras foram obtidas em cinco quadrantes de $1 \mathrm{~m}^{2}$, a cada $15 \mathrm{~m}$, e em cada uma das estações, coletadas com auxílio de espátula de pedreiro e acondicionadas em frascos opacos.

Em cada local, a esteira foi igualmente cortada verticalmente até atingir $5 \mathrm{~mm}$ de profundidade, com auxílio de bisturi cirúrgico. A constatação in loco da presença de uma estratificação levou à posterior individualização com o auxílio de microscópio estereoscópico. Cada estrato foi mensurado com o auxílio de paquímetro e as médias calculadas. As amostras foram armazenadas em potes opacos em solução de formol a 4\%, para análise posterior de cada uma das camadas.

Na fixação das cianobactérias o procedimento foi o mesmo (solução aquosa de formol a 4\%), com posterior tamponamento com bórax.

A análise taxonômica envolveu a confecção de 30 lâminas, divididas em números iguais entre frescas, semipermanentes e permanentes. Para a preparação das últimas, foi utilizado Bálsamo do Canadá para selamento e, nas semipermanentes, esmalte incolor. Os organismos foram observados quanto às características morfológicas e seguindo os padrões usuais. As medidas ao microscópio foram realizadas com auxílio de ocular micrométrica. Para cada espécie realizaram-se seis mensurações e obtiveram-se os valores médios, mínimos e máximos. Além disto, foram mensurados os seguintes parâmetros: diâmetro dos filamentos, diâmetro das colônias, diâmetro dos tricomas, espessura das bainhas, comprimento e largura das células.

\section{RESULTADOS}

A esteira coloforme melhor estudada distribui-se no fundo da lagoa Pitanguinha, em porções de inframaré, sendo raramente exposta ao ar. Externamente, apresenta inúmeras protuberâncias organizadas em fileiras (Figura 1B) e internamente, uma estruturação em lâminas de coloração variada.

O estrato superficial apresenta cores esverdeadas, devidas ao grande número de cianobactérias filamentosas, e possui uma espessura média de $3,4 \mathrm{~mm}$. O intermediário possui cores marrons, pela presença de matéria orgânica amorfa, abundantes cianobactérias cocóides, e uma espessura média de 2,62 mm. O mais profundo tem coloração vermelha, pela presença de bactérias sulfurosas púrpuras e cianobactérias cocóides, e 2,58 mm de espessura média. Distribuição semelhante foi encontrada por Silva e Silva (2002) para a lagoa Salgada, em áreas ao norte do Estado do Rio de Janeiro.

No conjunto, foram identificados 24 táxons de cianobactérias (ver Apêndice). No estrato verde, domina a família Chroococcaceae Nägeli, 1849, com 36,84\% das espécies; no marrom, a família Synechococcaceae Komárek \& Anagnostidis, 1995, com 33,34\% das espécies; e, no vermelho, mais uma vez as Chroococcaceae, com $38,46 \%$. As demais famílias observadas apresentaram os seguintes percentuais: Synechococcaceae Komárek \& Anagnostidis, 1995, 29,17\%, Phormidiaceae Anagnostidis \& Komárek, 1988, $12,5 \%$, Oscillatoriaceae Gomont, 1892, 8,35\%, Pseudanabaenaceae Anagnostidis \& Komárek, 1988, 4,16\%, Schizothricaceae Elenkin, 1934, 4,16\% e Entophysalidaceae, Geitler 1925, representando 4,16\%.

As espécies de forma esférica encontradas foram Aphanothece conglomerata, A. halophytica, A. marina, A. pallida, A. saxicola (Figura 2A), A. stagnina, Chroococcus membraninus, C. microscopicus, C. minimus, C. minor, $C$. minutus, C. obliteratus, C. prescottii, C. tenax, C. turgidus, Entophysalis granulosa e Gloeothece confluens. As filamentosas são Leptolyngbya tenuis, Lyngbya aestuarii, L. fragilis, Microcoleus chthonoplastes (Figura 2B), Schizothrix friesii, Spirulina subtilissima e S. subsalsa. A

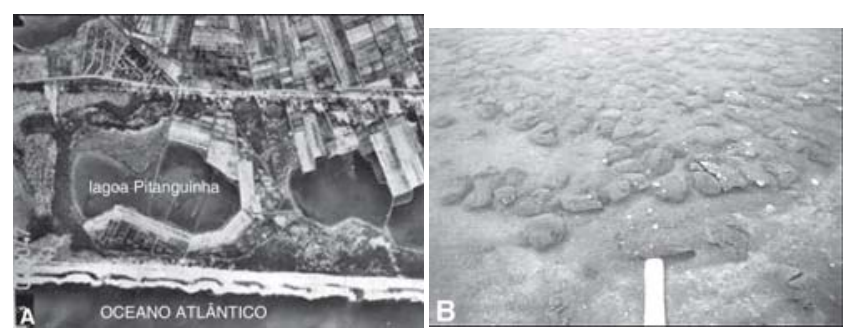

Figura 1. A, foto aérea da lagoa Pitanguinha (Brasil, 1976, escala 1:50.000); B, esteira microbiana coloforme submersa, de onde foram coletadas as amostras analisadas.

Figure 1. A, Air photo of the Pitanguinha Lagoon (Brasil, 1976, scale 1:50,000); B, Coloform microbial mat on the lagoon's bottom, from where the analyzed samples came from.

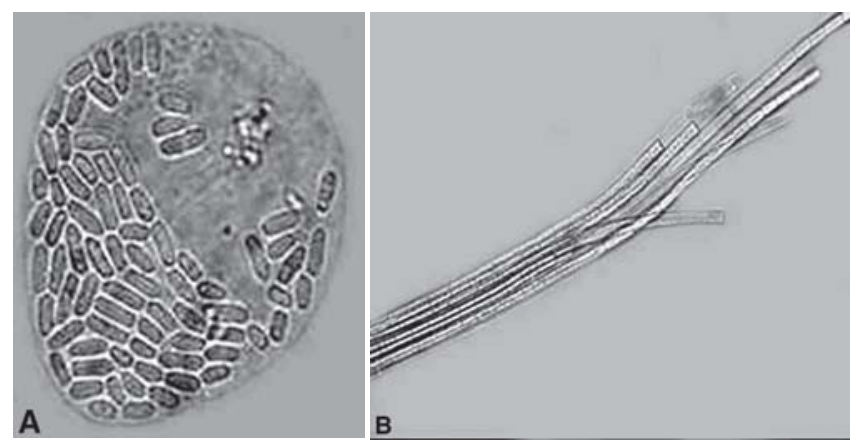

Figura 2. Cianobactérias presentes na esteira microbiana coloforme da lagoa Pitanguinha. A, Aphanothece saxicola Nägeli 1849, esférica; B, Microcoleus chthonoplastes (Thuret) Gomont 1892, filamentosa.

Figure 2. Cyanobacteria from the coloform macrobial mat of the Pitanguinha Lagoon. A, Aphanothece saxicola Nägeli 1849, spherical form; B, Microcoleus chthonoplastes (Thuret) Gomont 1892, filamentous form. 
distribuição das espécies ao longo dos estratos é mostrada na Tabela 1, e das famílias, na Figura 3.

As formas distribuem-se ainda de modo distinto, de acordo com as camadas que compõem a esteira microbiana. Nas camadas superficiais, por exemplo, os principais gêneros de cianobactérias são Microcoleus Gomont, Lyngbya (Agardh) Gomont, Spirulina (Turpin) Gomont e Phormidium (Kützing) Gomont, uma condição similar àquela identificada por Urmeneta \& Navarrete (2000), em esteiras microbianas do delta do rio Ebro (Espanha).

Três formas presentes nas esteiras da lagoa Pitanguinha são características de ambientes hipersalinos. Lyngbya aestuarii, caracteriza o tipo de esteira tufada e aparece associada ao substrato arenoso, como já havia sido apontado por Santelli (1988), em sedimentos da lagoa Vermelha. Microcoleus chthonoplastes, espécie responsável pelas esteiras em placas, é a espécie mais abundante na lagoa Pitanguinha e foi igualmente identificada em esteiras microbianas das salinas em San-ya, Ilha de Hai-nan, China (Zhang \& Hoffmann, 1992). Segundo Baeta Neves \& Casarin (1990), também Aphanothece stagnina é associada a este tipo de ambiente.

A análise por estrato demonstrou uma diminuição gradativa da quantidade de cianobactérias, bem como a substituição das espécies filamentosas por esféricas, atestando um maior grau de maturação, quando se vai das camadas mais superficiais para as mais internas. Este resultado igualmente está de acordo com estudos prévios realizados em áreas próximas (Carvalhal, 2003). A substituição das formas filamentosas por esféricas deve-se a fatores como intensidade luminosa e temperatura, que agem como limitantes para as cianobactérias. Conforme aumenta a profundidade, diminuem de intensidade, causando a morte das filamentosas e o aumento das esféricas, mais adaptáveis e esta condição (Carvalhal, 2003).

Microgastrópodes, ostracodes e bivalves foram encontrados associados às esteiras e constituem mais uma fonte de carbonato de cálcio. Sua capacidade ainda de servir de substrato para as cianobactérias já havia sido verificada anteriormente por Silva e Silva et al. (1999). Já a presença de laminações, reflete o modo de crescimento das cianobactérias e depende da forma como ocorre a captação e a precipitação do carbonato de cálcio (Silva e Silva et al., 2004). A variação em sua espessura demonstra uma relação direta com os períodos de cheia e vazante da lagoa, caracterizando, assim, períodos mais secos ou mais úmidos (Silva e Silva, 2002).

\section{CONCLUSÕES}

A variedade de formas presentes nas esteiras microbianas da lagoa Pitanguinha sugere uma plasticidade morfológica e uma boa capacidade de sobrevivência nos ambientes estressantes onde se desenvolveram, sujeitos a um clima

Tabela 1. Distribuição das cianobactérias ao longo dos estratos da esteira coloforme na lagoa Pitanguinha: A, estrato verde; B, estrato marrom; C, estrato vermelho.

Table 1. Cyanobacterial distribution in the beds of the Pitanguinha Lagoon coloform mat. A, green bed; B, brown bed; C, red bed.

\begin{tabular}{|c|c|c|c|}
\hline $\begin{array}{ll} & \text { Estratos } \\
\text { Táxons } & \end{array}$ & $\mathbf{A}$ & B & C \\
\hline $\begin{array}{l}\text { Aphanothece } \\
\text { conglomerata }\end{array}$ & $\mathrm{X}$ & $\mathrm{X}$ & $\mathrm{X}$ \\
\hline Aphanothece halophytica & $x$ & & \\
\hline Aphanothece marina & $x$ & $x$ & $x$ \\
\hline Aphanothece pallida & $x$ & & \\
\hline Aphanohthece saxicola & $x$ & $x$ & $x$ \\
\hline Aphanothece stagnina & $x$ & $x$ & $x$ \\
\hline $\begin{array}{l}\text { Chroococcus } \\
\text { membraninus }\end{array}$ & $x$ & & $x$ \\
\hline $\begin{array}{l}\text { Chroococcus } \\
\text { microscopicus }\end{array}$ & $x$ & $x$ & \\
\hline Chroococcus minimus & $x$ & $x$ & \\
\hline Chroococcus minor & $x$ & $x$ & $x$ \\
\hline $\begin{array}{l}\text { Chroococcus minutus } \\
\text { Chroococcus obliteratus }\end{array}$ & & $x$ & $\begin{array}{l}x \\
x\end{array}$ \\
\hline Chroococcus prescottii & $x$ & & \\
\hline Chroococcus tenax & $x$ & & \\
\hline Chroococcus turgidus & $x$ & $x$ & \\
\hline $\begin{array}{l}\text { Entophysalis granulosa } \\
\text { Gloeothece confluens }\end{array}$ & & & $\begin{array}{l}x \\
x\end{array}$ \\
\hline Leptolyngya tenuis & $x$ & $x$ & $x$ \\
\hline $\begin{array}{l}\text { Lyngbya aestuarii } \\
\text { Lyngbya fragilis }\end{array}$ & $x$ & & $\begin{array}{l}x \\
X\end{array}$ \\
\hline $\begin{array}{l}\text { Microcoleus } \\
\text { chthonoplastes }\end{array}$ & $x$ & & $x$ \\
\hline Schizothrix friesii & $x$ & $x$ & $x$ \\
\hline Spirulina subsalsa & $x$ & $x$ & \\
\hline Spirulina subtilissima & $x$ & $x$ & \\
\hline TOTAL & 19 & 13 & 15 \\
\hline
\end{tabular}
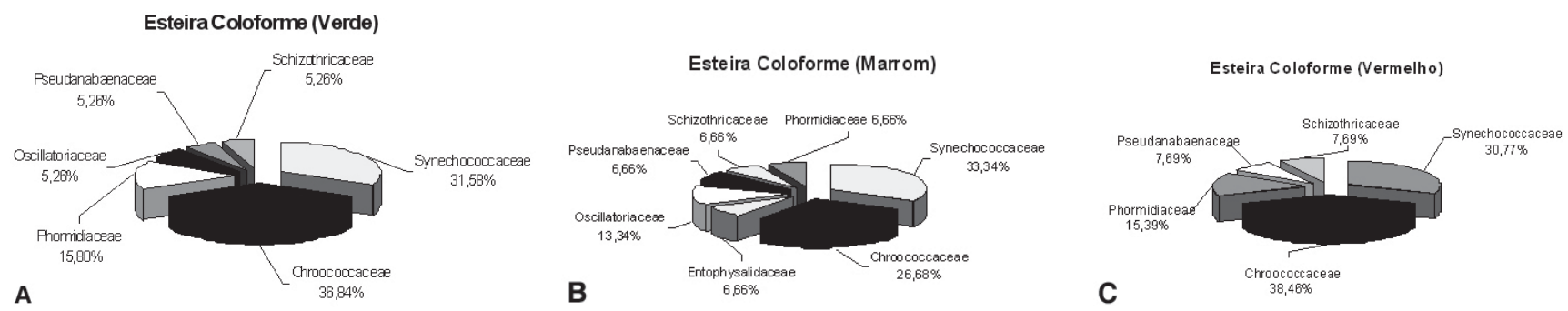

Figura 3. Distribuição das famílias de cianobactérias nos estratos da esteira coloforme da lagoa Pitanguinha: A, estrato esverdeado; B, estrato marrom; C, estrato vermelho.

Figure 3. Family distribution in the Pitanguinha Lagoon coloform mat: A, green bed; B, brown bed; C, red bed. 
caracterizado por déficit de chuva e a variações do nível do mar provocadas pelas marés.

As variações de coloração verificadas nos estratos da esteira devem-se às variações em sua composição, em termos das formas presentes, à profundidade e à intensidade de luz.

Estes dados podem ser úteis para o estudo de outras formações estromatolíticas e esteiras microbianas e, especialmente, de grande valia nas extrapolações para o registro fóssil, onde os diferentes tipos de esteiras e sua estruturação em lâminas podem ser utilizados na avaliação das variações de salinidade do meio, profundidade das águas e variações eustáticas do nível do mar pretéritas.

\section{AGRADECIMENTOS}

Agradecemos ao Serviço Geológico do Brasil - CPRM pela cessão da fotografia aérea utilizada na Figura 1A.

\section{REFERÊNCIAS}

Abreu, P.C. \& Odebrecht, C. 2000. Microorganismos em lagoas costeiras: um estudo de caso do estuário da lagoa dos Patos (RS). In: F.A. Esteves \& L.D. Lacerda (eds.) Ecologia de restingas e lagoas costeiras, Editora da UFRJ, p. 215-230.

Altermann, W. \& Kazmierezak, J. 2003. Archean microfossils: a reappraisal of early life on Earth. Research in Microbiology, 154:611-617.

Anjos, A.P.A. 1999. Caracterização da sedimentação atual de lagoas costeiras do litoral do Estado do Rio de Janeiro para fins de calibração de acordo com as variações paleoambientais e paleoclimáticas. Programa de Pós-graduação em Geoquímica, Universidade Federal Fluminense, Dissertação de Mestrado, $133 \mathrm{p}$.

Baeta Neves, M.H.C. \& Casarin, A.J. 1990. As cianofíceas das salinas de Cabo Frio - Brasil. Acta Biologica Leopoldensia, 12(1):99-123.

Barroso, L.V. 1987. Diagnóstico ambiental da lagoa de AraruamaRJ. Boletim da Fundação Brasileira de conservação da Natureza, 22:30-65.

Batchelos, M.T.; Burne, R.V.; Henry, B.I. \& Watt, S.D. 2000. Deterministic KPZ model for stromatolite laminae. Physica, 282:123-136.

Brasil-Ministério da Aeronáutica. 1976. Fotografia aérea da lagoa Pitanguinha, Região dos Lagos, Rio de Janeiro, Brasil, foto 113/ 000047, escala 1:50.000.

Carvalhal, S.B.V. 2003. Bioestratificação cianobacteriana das esteiras microbianas recentes da lagoa Vermelha, Rio de Janeiro, Brasil. Programa de Graduação em Ciências Biológicas, Universidade Federal do Estado do Rio de Janeiro, Monografia de Bacharelado, $80 \mathrm{p}$.

Fonseca, M.C. 2002. Avaliação de indice de risco ecológico potencial para a contaminação por metais pesados. Caso das lagoas do leste fluminense. Programa de Pós-graduação em Geoquímica, Universidade Federal Fluminense, Dissertação de Mestrado, 116 p.

Golubic, S. 1976. Organismis that build stromatolites. In: M.R. Walter (ed.) Stromatolites, Elsevier Science, p.113-126.

Lemos, R.M.T. 1996. Estudo das fácies deposicionais e das estruturas estromatolíticas da lagoa Salgada, Rio de Janeiro. Programa de Pós-graduação em Geofísica Marinha Geoquímica,
Universidade Federal Fluminense, Dissertação de Mestrado, $126 \mathrm{p}$.

Lopes, C.E.A. 1988. Estudo em laboratório de absorção do metal cobre pelo tapete de algas bênticas da lagoa Vermelha- Município de Araruama- Rio de Janeiro. Programa de Pós-graduação em Geoquímica, Universidade Federal Fluminense, Dissertação de Mestrado, 56 p.

Nascimento, S.M. 1999. Algas azuis. Recife, Universidade Federal de Pernambuco e ITEP, 28 p.

Noffke. N.; Gerdes, G.; Klenke, T. \& Krumbein, W.B. 2001. Microbially induced sedimentary structures - a new category within the classification of primary sedimentary structures. Journal of Sedimentary Research, 71(5):649-656.

Patchineelan, S.R. \& Naidu, A.S. 1995. Estudos bioquímicos numa lagoa costeira: uma interpretação ambiental. In: CONGRESSO BRASILEIRO DE GEOQUÍMICA E CONGRESSO DE GEOQUÍMICA DOS PAIISES DE LÍNGUA PORTUGUESA, 5, 1995. Resumos, Niterói, UFF, p. 245-253.

Primo, P.B.S. \& Bizerril, C.R.S.F. 2002. Lagoa de Araruama. Perfil ambiental do maior ecossistema lagoar hipersalino do mundo. Rio de Janeiro, SEMADS, p. 33-35.

Santelli, R.C.L. 1988. Estudos de isótopos estáveis em sedimentos carbonáticos da lagoa Vermelha, Rio de Janeiro. Programa de Pós-graduação em Geoquímica, Pontifícia Universidade Católica, Tese de Doutorado, 85 p.

Schieber, J. 1998. Possible indicators of microbial mat deposits in shales and sandstones: examples from the Mid-Proterozoic Belt Supergroup, Montana, USA. Sedimentary Geology, 120:105-124.

Silva e Silva, L.H. 2002. Contribuição ao conhecimento da composição microbiana e química das estruturas estromatolíticas da lagoa Salgada, Quaternário do Rio de Janeiro, Brasil. Programa de Pós-graduação em Geologia, Universidade Federal do Rio de Janeiro, Tese de Doutorado, 176 p.

Silva e Silva, L. H.; Senra, M. C. E. \& Srivastava, N.K. 1999. Composição ficológica dos testemunhos dos estromatólitos estratiformes e esteira algal na lagoa Salgada, Rio de Janeiro, Brasil. In: CONGRESSO DA ASSOCIAÇÃO BRASILEIRA DE ESTUdos DO QUATERNÁRIO, 7, 1999. Anais, Porto Seguro, CD-ROM.

Silva e Silva, L.H.; Srivastava, N.K.; Carvalhal, S.B.V.; Alves, S.A.P.M.N.; Faruolo, T.C.L M; Damazio, C.M.; Shimizu, V.T.A. 2002. Distinção entre as estruturas calcárias e estágios de desenvolvimento de construções estromatolíticas na lagoa Salgada, Rio de Janeiro, Brasil. In: CONGRESSO BRASILEIRO DE GEOLOGIA, 41, 2002. Resumos, Recife, SBG, p. 367.

Silva e Silva, L.H.; Senra, M.C.E.; Faruolo, T.C.L.M.; Carvalhal, S.B.V.; Alves, S.A.P.M.N.; Damazio, C.M.; Shimizu, V.T.A. \& Iespa, A.A.C. 2003a. Distinção entre as esteiras microbianas da lagoa Pitanguinha, Quaternário do Rio de Janeiro, Brasil. In: CONGRESSO BRASILEIRO DE PALEONTOLOGIA, 18, 2003. Anais, Brasília, SBP, p. 271.

Silva e Silva, L.H.; Damazio, C.M. \& Barros, E.S., 2003b. Identificação da composição das esteiras estromatolíticas poligonais da lagoa Pitanguinha, Rio de Janeiro, Brasil. Paleontologia em Destaque, 44:23.

Silva e Silva, L.H.; Senra, M.C.E.; Farulo, T.C.L.M.; Carvalhal, S.B.V.; Alves, S.A.P.M.N.; Damazio, C.M.; Shimizu, V.T.A.; Santos, R.C. \& Iespa, A.A.C. 2004. Composição Paleobiológica e Tipos Morfológicos das Construções Estromatolíticas da lagoa Vermelha, RJ, Brasil. Revista Brasileira de Paleontologia, 7(2):193-198. 
Turcq, P.F.M. 2000. Impact of low salinity year on the metabolism of a hipersaline coastal lagoon (Brasil). Hidrobiologia, 429:133-140.

Turcq, B.; Martin, L.; Flexor, J.M.; Suguio, K.; Pierre, C.; Tasayco Ortega, L. 1999. Origin and evolution of the Quaternary coastal plain between Guaratiba and Cabo Frio, State of Rio de Janeiro, Brazil. In: B. Knoopers, E.D. Bidone \& J.J. Abraão (eds.) Environmental geochemistry of coastal lagoon systems of Rio de Janeiro, Brazil. Rio de Janeiro, Universidade Federal Fluminense, p. 25-46 (Série Geoquímica Ambiental).

Urmeneta, J. \& Navarrete, A. 2000. Mineralogical composition and biomass studies of the microbial mats sediments from the Ebro Delta, Spain. International Microbiology, 3:97-101.

Van Germerden, H. 1993. Microbial mats: a joint venture. Marine Geology, 113:3-25.

Vasconcelos, C.O. 1988. Sedimentologia e geoquímica da lagoa Vermelha-um exemplo de formação e diagênese de carbonatos. Programa de Pós-graduação em Geoquímica, Universidade Federal Fluminense, Dissertação de Mestrado, 63 p.

Weber, W. 2001. Ambiente das Águas no Estado do Rio de Janeiro. Rio de Janeiro, SEMADS, 230 p.

Zhang, Y \& Hoffmann, L. 1992. Blue-green algal mat of the Salinas in San-ya, Hai-nan Island (China): structure taxonomic composition and implications for the interpretation of Precambrian stromatolites. Precambrian Research, 56:275-290.

Received in January, 2006; accepted in March, 2006.
Apêndice. Lista das cianobactérias identificadas na lagoa Pitanguinha.

Appendix. List of identified cianobacteria to Pitanguinha Lagoon.

\section{Formas esféricas}

Aphanothece conglomerata Rich, 1932

A. halophytica Frémy, 1933

A. marina (Ercegovi) Komárek \& Anagnostidis, 1995

A. pallida (Kützing) Rabenhorst, 1863

A. saxicola Nägeli, 1849

A. stagnina (Sprengel) A. Braum, 1863

Chroococcus membraninus (Meneghini) Nägeli, 1849

C. microscopicus Komárková-Legnerová \& Cronberg, 1994

C. minimus (Keissler) Lemmermann, 1904

C. minor (Kützing) Nägeli, 1849

C. minutus (Kützing) Nägeli, 1849

C. obliteratus Richter, 1886

C. prescottii Drouet \& Daily, 1942

C. tenax (Kirchner) Hieronymus, 1892

C. turgidus (Kützing) Nägeli, 1849

Entophysalis granulosa Kützing, 1843

Gloeothece confluens Nägeli, 1849

\section{Formas filamentosas}

Leptolyngbya tenuis (Gomont) Anagnostidis \& Komárek, 1988 Lyngbya aestuarii (Liebman) Gomont, 1892

Lyngbya fragilis (Meneghini) Compère, 1974

Microcoleus chthonoplastes (Thuret) Gomont, 1892

Schizothrix friesii (Agardh) Gomont, 1892

Spirulina subtilissima Kützing, 1843

S. subsalsa (Gersted) Gomont, 1892 